\title{
Euler Characteristic of Polyhedral Graphs
}

Atena Pîrvan-Moldovan, Mircea V. Diudea*

\author{
Department of Chemistry, Faculty of Chemistry and Chemical Engineering, Babes-Bolyai University, 400028 Cluj, Romania \\ * Corresponding author's e-mail address: diudea@chem.ubbcluj.ro \\ RECEIVED: October 5, 2016 * REVISED: November 7, 2016 * ACCEPTED: November 11, 2016
}

THIS PAPER IS DEDICATED TO PROF. NENAD TRINAJSTIĆ ON THE OCCASION OF HIS 80 ${ }^{\text {TH }}$ BIRTHDAY

\begin{abstract}
Euler characteristic is a topological invariant, a number that describes the shape or structure of a topological space, irrespective of the way it is bent. Many operations on topological spaces may be expressed by means of Euler characteristic. Counting polyhedral graph figures is directly related to Euler characteristic. This paper illustrates the Euler characteristic involvement in figure counting of polyhedral graphs designed by operations on maps. This number is also calculated in truncated cubic network and hypercube. Spongy hypercubes are built up by embedding the hypercube in polyhedral graphs, of which figures are calculated combinatorially by a formula that accounts for their spongy character. Euler formula can be useful in chemistry and crystallography to check the consistency of an assumed structure.
\end{abstract}

Keywords: Euler characteristic, polyhedral graph, hypercube, map operation.

\section{INTRODUCTION}

\subsection{Euler Characteristic}

$\mathbf{E}^{2}$ ULER characteristic ${ }^{[1]}$ is a topological invariant, a single number that describes the shape of a structure regardless of its tiling; it is denoted by $\chi$ (small Greek "chi") and makes the subject of study in algebraic topology, polyhedral combinatorics, crystallography and in many other mathematical fields. ${ }^{[2]}$ Euler characteristic, named after Leonhard Euler, was originally defined for polyhedra and used to check the consistency of a proposed structure.

Any polyhedron can be represented in the plane by a 3-connected planar graph (also called a polyhedral graph). The number of vertices $v$, edges $e$, and faces $f$ of a convex polyhedron are related by the Euler's polyhedron formula

$$
v-e+f=\chi,
$$

in which case $\chi=2$, i.e., the Euler characteristic for the sphere. The surface of nonconvex polyhedra may have various $\chi$-values (see below).

There are many proofs of Euler's formula, ${ }^{[2-7]}$ as many definitions and implications in various mathematical fields of the Euler characteristic were explored (these are, however, out of the aim of this study).
Euler characteristic of a closed orientable surface can be calculated from its genus $g$ (the number of tori in a connected sum decomposition of the surface, or the number of handles or holes an object has) by the Poincaré formula

$$
v-e+f=\chi=2(1-g) \text {. }
$$

Euler characteristic of a closed non-orientable surface can be calculated from its non-orientable genus $k$ (the number of real projective planes in a connected sum decomposition of the surface or the number of cross-caps needed to be attached to the sphere to make it homeomorphic to that surface) as

$$
\chi=2-k \text {. }
$$

A surface is orientable, when it has two sides, or it is non-orientable, when it has only one side, like the Möbius strip.

In differential geometry, the Gauss-Bonnet theorem $^{[8]}$ states that the geometry of a surface $S$ (as described by Gaussian curvature $K$ ) is related to its topology (as expressed by Euler characteristic)

$$
\int_{S} K d S=2 \pi \chi
$$

Positive/negative $\chi$ - values indicate positive/negative curvature of the polyhedral structure. 
A discrete analog of the Gauss-Bonnet theorem is due to Descartes; ${ }^{[9]}$ it shows that the overall angular defects (i.e., disclinations), measured in full circles, is the Euler characteristic of the polyhedron.

$$
\sum_{p} \varphi_{p}=2 \pi \chi
$$

More recently, a "combinatorial curvature" which parallels "Gaussian curvature" of the embedding surface, was proposed. ${ }^{[10,11]}$

Euler characteristic can be calculated for general surfaces as the alternating sum of figures of dimension/rank $k$

$$
\chi=f_{0}-f_{1}+f_{2}-f_{3}+\ldots,
$$

by finding a polygonization of the surface (i.e., a description as a CW-complex ${ }^{[12,13]}$ ).

\subsection{Operations on Maps}

Structures discussed hereafter are represented by simple, non-directed graphs. Their design was made by operations on maps, merely applied on the Platonic solids: tetrahedron $(T)$, cube $(C)$, octahedron (O), dodecahedron (D) and icosahedron (I). A map is a discretized closed surface, i.e., a polyhedron $\mathrm{P}$, while the operations on maps are topological modifications of a parent map. The symmetry of parents is preserved by running these operations. Several operations are known (under various names/symbols) and currently used to decorate/transform a given mathematical object: dual $d$; medial $m$; truncation $t$; leapfrog $l$; polygonal $p_{k}$, snub, etc. The reader is invited to consult some publications in this respect. ${ }^{[14-16]}$ In the following, only the most used map operations are shortly described.

Dual $d(P)$ is obtained by putting a point in the center of each face of a polyhedron $P$, next joining two such points if their corresponding faces share a common edge; it is the Poincaré dual. Vertices of $d(P)$ represent faces in the parent polyhedron and, vice versa. Dual of the dual returns the original polyhedron: $d(d(\mathrm{P}))=\mathrm{P}$. Tetrahedron is self-dual while the other Platonics form pairs: $d(\mathrm{C})=0 ; d(\mathrm{D})=\mathrm{I}$. Dualization is an operation in any dimensions and can be written, with the Schläfli symbols, ${ }^{[17]}$ as the reverted polytope figure type: $\{a, b, c, \ldots, y, z\}$ becomes $\{z, y, \ldots, c, b, a\}$ in the dual polytope. In general, the facets of a polytope's dual will be the topological duals of the polytope's vertex figures.

A Petrie dual (or Petrial) is also known; ${ }^{[18]}$ it is a map having vertices and edges of the original polyhedron and whose faces are the set of Petrie polygons. The Petrie polygon of a polyhedron is a skew polygon of which every two consecutive edges (but not three) belong to one of the faces of the parent polygon. For the Platonic polyhedra ( $T$, C, O, D and I), the Petrie polygons are: 3 squares, 4 hexagons, 4 hexagons, 6 decagons and 6 decagons, respectively.
Petrie polygon for a regular polytope of $n$ dimensions is a skew polygon such that every $(n-1)$ consecutive sides (but not $n$ ) belong to one of the facets of the parent polytope. Petrials are useful in visualizing the symmetric structure of higher-dimensional regular polytopes.

Truncation $t(P)$ is achieved by cutting off the neighborhood of each vertex by a plane close to the vertex, such that it intersects each edge incident in the vertex. The resulted truncated map (i.e., polyhedron) is always a three-connected one. The truncated polyhedron is of the type $\{2 e$, $3 e, e+\chi\}$, where $e$ denotes the number of edges in the parent object while the numbers within brackets refer subsequently to the vertices, edges and faces of the truncated transform; it works in polytopes of any dimension, creating a new facet instead of each vertex. This was the main operation used by Archimedes in building its well-known 13 solids while the term Archimedean solids was given by Kepler.

Medial $m(P)$ is obtained by pairwise joining the midpoints of parent edges if the pair edges span an angle. Medial is always a 4-valent graph, symmetric between the parent and its dual, that is $m(P)=m(d(P))$. The figure type of the transformed polyhedron is: $\{e, 2 e, e+\chi\}$.This operation rotates the parent $s$-gonal faces by $\pi / s$. By medial, edges of the parent polyhedron are reduced to a point; this property can be used in topological analysis of edges. This operation is also known as the rectification or even ambo (Conway notation ${ }^{[16]}$ ). Applying twice $\mathrm{mm}$ (medial of a medial) is the same as expansion operation of Conway or Johnson's cantellation operation. ${ }^{[14]}$

Leapfrog $I(P)$ is a composite operation ${ }^{[19-21]}$ that can be written as: $I(P)=t(d(P))$. It rotates the parent $s$-gonal faces by $\pi / s$. In a three-connected polyhedron, the transformed polyhedron is of the type: $\{2 e, 3 e, e+\chi\}$. Note that, in the transform $I(P)$, the vertex degree is always 3 , since this operation involves a truncation that provides a trivalent lattice.

Polygonal $p_{k}(P)$ operation is achieved by adding a new vertex in the center of each face of a polyhedral graph, next put $k-3$ points on the boundary edges. Connect the central point with one vertex on each edge (the endpoints included): the parent face will be covered by triangles $(k=3)$, squares $(k=4)$ and pentagons $(k=5)$, respectively. The transformed polyhedron is of the type: $\{(k-2) e+\chi, k e$, $2 e\}$ [21] $^{[21}$

Snub is the dual of $p_{5}$ operation: ${ }^{[21]} s(P)=d\left(p_{5}(P)\right.$ and $s(P)=$ $s(d(P))$. The snub polyhedron is of the type: $\{2 e, 5 e, 3 e+\chi\}$. In case $P=\mathrm{T}$, the snub is the icosahedron: $s(\mathrm{~T})=1$.

The paper is organized as follows. After an introduction to Euler characteristic definitions, the operations on maps are described. The main results refer to pairs of map 
operations (Section 2), with some details on the truncation operation and its use in transforming the cubic crystal network and the hypercube (Section 3). A further Section 4 introduces the spongy hypercubes, built up by embedding the hypercube in polyhedra, on which Euler characteristic is calculated. Conclusions and references will close the article.

\section{PAIRS OF MAP OPERATION}

The alternating sum of figures of rank $k$ of an $n$-polytope is equal to the Euler charasteristic $\chi$, as shown in (6). Next, according to the Poincare formula (2), $\chi=2(1-g)$, with $g$ being the genus of the surface in which the polyhedral graph is embedded; in case of the sphere, $\chi=2$ and $g=0$; for the torus, $\chi=0$ and $g=1$, while in case $\chi<0$, the surface shows a negative curvature.

Theorem 1. Let $\{v, e, f\}$ and $\left\{n_{1} e+\delta, n_{2} e, n_{3} e\right\}$ be types of $a$ parent polyhedron and its derivative polyhedron $o(P)$ (obtained by a map operation $o$ ). Also, let both $P$ and $o(P)$ have the same Euler characteristic $\chi$. Then, $\delta=\chi$ if and only if $\left(n_{1}+n_{3}\right)=n_{2}$.

Proof. Assuming that the transformed polyhedron $o(P)$ has the same $\chi$-characteristic as the parent polyhedron $P$, the Euler formula (1) can be re-written, in case of $o(P)$, as:

$$
\left(n_{1} e+\delta\right)-\left(n_{2} e\right)+\left(n_{3} e\right)=\chi,
$$

and rearrange it as:

$$
e\left(n_{1}-n_{2}+n_{3}\right)+\delta=\chi .
$$

It is clear that equality $\delta=\chi$ holds only if

$$
\left(n_{1}+n_{3}\right)=n_{2} .
$$

Condition (9) is both a necessary and sufficient condition for Theorem 1 to be true.

Corollary 1 (2). The dual of the generalized transform $d(o(P))$ will have the type: $\left\{n_{3} e, n_{2} e, n_{1} e+\chi\right\}$. This comes out from the property of Schläfli symbol that its reversal gives the symbol of the dual polyhedron.

Corollary 1 (3). Difference in the number of vertices of polyhedral graphs transformed by selected pairs of map operations, $O_{1}$ and $o_{2}$, equals the Euler characteristic of the embedding surface (Table 1$):\left|V\left(o_{1}(P)\right)\right|-\left|V\left(o_{2}(P)\right)\right|=\chi$. Difference may be done with respect to the polyhedron faces but the counting of faces is more expensive. Such pairs of map operations will be illustrated in the following (Figures 1 to 4). The figure count for the structures in Figures 2 to 4 is given in Table 2. Note that the number of points/atoms suffixes the name of structures.

\begin{tabular}{|c|c|c|}
\hline Operation & $O(P)$ & $d(o(P))$ \\
\hline leapfrog (I) & $\{2 e, 3 e, e+\chi\}$ & $\{e+\chi, 3 e, 2 e\}$ \\
\hline medial $(m)$ & $\{e ; 2 e ; e+\chi\}$ & $\{e+\chi ; 2 e ; e\}$ \\
\hline medial^ $\wedge^{\wedge}=1,2, . .\left(m^{\wedge} k\right)$ & $\begin{array}{c}\left\{2^{\wedge}(k-1) e, 2\left(2^{\wedge}(k-1)\right) e\right. \\
\left.2^{\wedge}(k-1) e+\chi\right\}\end{array}$ & - \\
\hline$p_{4}=d m^{\wedge} 2$ & $\{2 e+\chi, 4 e, 2 e\}$ & $\{2 e, 4 e, 2 e+\chi\}=m^{\wedge} 2$ \\
\hline truncation $(t)$ & $\{2 e, 3 e, e+\chi\}$ & $\{e+\chi, 3 e, 2 e\}$ \\
\hline \multirow[t]{7}{*}{$\left|V\left(o_{1}(P)\right)\right|-\left|V\left(o_{2}(P)\right)\right|=\chi$} & $o_{1}$ & $\mathrm{O}_{2}$ \\
\hline & $d m$ & $m$ \\
\hline & $d l$ & $m$ \\
\hline & $d t$ & $m$ \\
\hline & $p_{4}$ & $d\left(p_{4}\right)=m m$ \\
\hline & $p_{4}$ & 1 \\
\hline & $p_{4}$ & $t$ \\
\hline $\begin{array}{c}|V(t(P))|=|V(/(P))|= \\
|V(m m(P))|=2|V(m(P))|\end{array}$ & (different edges and faces) & \\
\hline$\left|V\left(p_{4}(P)\right)\right|=|V(d m m(P))|$ & (identical edges and faces) & \\
\hline
\end{tabular}

Table 1. Operations on maps and pair of operations providing the Euler characteristic.

\section{TRUNCATION OPERATION}

Let $H=(V, E)$ be a simple graph with the vertex set $V$ and edge set $E$. A perfect matching $M$ of a simple graph $H$ having an even number of vertices $|V(H)|=2 h$ is a subset $E(M) \subset E(H)$ of its $h$ pairwise nonincident edges covering all vertices of $H$. Similarly, a cycle cover $C$ is a set of disjoint cycles covering all vertices of $H, E(C) \subset E(H)$. Note that, in general, not every graph has $M$ and/or $C$. In a simple cubic graph, the two subsets of edges are dijoint and complementary: $E(M) \cap E(C)=0$ and $E(M) \cup E(C)=E(H)$, meaning their union contains all the edges of $H$. These notions are illustrated in Figure 5.

There is a result from Ref. [22], stating that: a necessary and sufficient condition for a simple cubic graph $G$, without quadrilaterals, to be isomorphic to the truncation $t(H)$ of a simple cubic graph $H$ is possessing by $G$ a cyclic cover $C$ all whose components are triangles (see Figure 5 ).

The above result may be extended to a simple graph having all the vertices of degree $d$ : the cyclic cover will consist of disjoint $d$-cycles. If the graph possesses vertices of different degree $d_{k}$, the cyclic cover will be the union of $d_{k}$ cycles.

Truncation may be seen as a defect occurring in a crystal network, like that envisaged in Figure 6 . While the full realization of truncation leads to a uninodal (i.e., single atom type) net, the defects induce a variety of atom types (Figure 6, right). RSI[23] was calculated by embedding the 8unit structures in a larger corresponding nets. 


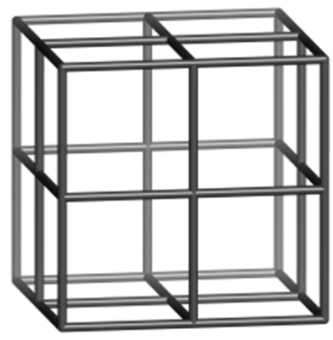

$p_{4}(\mathrm{C}) .26 ;\left(8\left(4^{\wedge} 3\right) \cdot 18\left(4^{\wedge} 4\right)\right)$

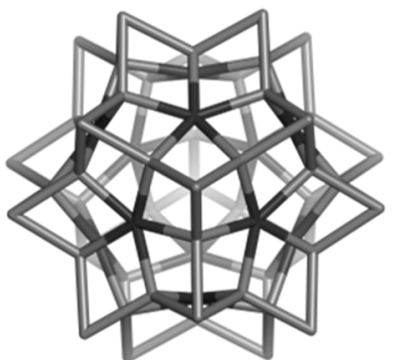

$p_{4}(\mathrm{D}) \cdot 62\left(12\left(4^{\wedge} 5\right) \cdot 30\left(4^{\wedge} 4\right) \cdot 20\left(4^{\wedge} 3\right)\right)$

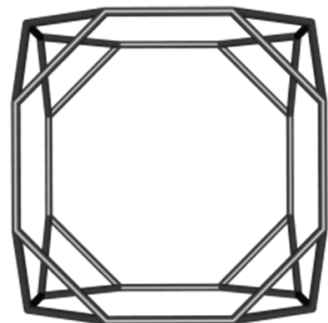

$t(C) .24 ;\left(3.8^{\wedge} 2\right)$

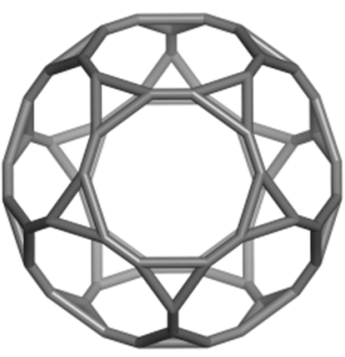

$t(\mathrm{D}) .60$

(3.10.10)

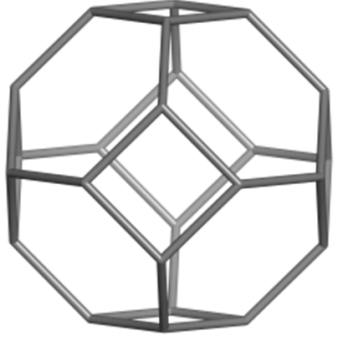

I(C). $24 ;\left(4.6^{\wedge} 2\right)$

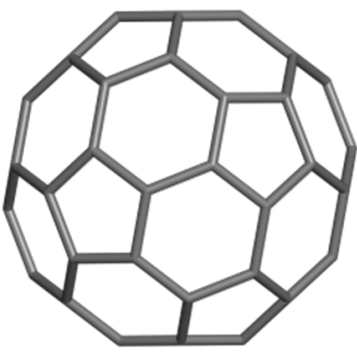

I(D). $60=\mathrm{C}_{60}$ (5.6.6)

Figure 1. Operations applied on Cube C (top) and Dodecahedron D (bottom): $o_{1}=p_{4}$ (left); $o_{2}=t$ (truncation, middle); and $o_{2}=I$ (leapfrog, right); vertex number differece: $\chi=2$ (Euler characteristic of the sphere).

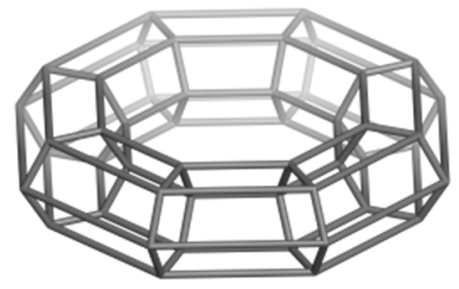

H6.8.48 $\left\{4^{4}\right\}$

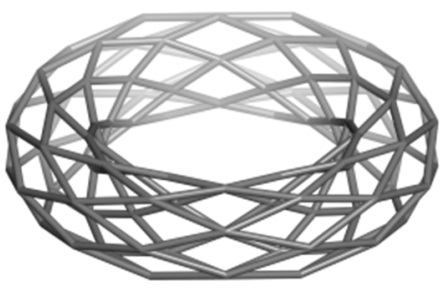

$d m(H 6.8) .96\left\{4^{4}\right\}$

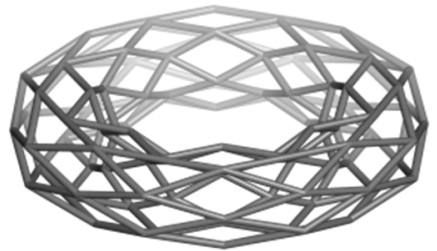

$m(\mathrm{H} 6.8) .96\left\{4^{4}\right\}$

Figure 2. Operations acting on a square-tiled torus (left): $o_{1}=d m$ (dual of medial, middle) and $o_{2}=m$ (right); vertex number differece: $\chi=0$ (Euler characteristic of the torus).

Counting the rings around any atom in such a transformed net, as the ring signature index RSI, recently proposed by us, ${ }^{[23]}$ could be a useful tool in theoretical crystallography.

Let now focus attention on the hypercube $Q_{n}$; the truncation replaces any parent vertex in $Q_{n}$ by a simplex $S_{n-1}$ (Ref. [24]) and these all trigonal substructures are disjoint while their union will cover all the vertices in the truncated transform $t\left(Q_{n}\right)$.

Squares $4(2)$ are changed by octagonal $8(2)$ faces while cubes are changed by truncated cubes (Figures 7 and 8 ).

The number of these simplices equals the number of vertices in $Q_{n}:\left|V\left(Q_{n}\right)\right|=2^{\wedge} n$; the number of vertices in each symplex equals $n$ (see Table 3 ). In the above structures, $n$ denotes the dimension or better the rank, ${ }^{[25]}$ since we refer here to shapes rather than to geometric polytopes. Figure count in the hypercube and its truncated transforms is detailed in Table 3. The number of $k$-facets $t\left(Q_{k}\right)$ is counted by adding to $Q_{k}$ (that equals the number of substructures with $8(2)$ faces) the number of facets of the corresponding simplex, as:

$Q_{n}^{k}=2^{n-k}\left(\begin{array}{c}n \\ k\end{array}\right) ; S_{n}^{k}=\left(\begin{array}{c}n+1 \\ k+1\end{array}\right) ; t\left(Q_{n}^{k}\right)=Q_{n}^{k}+S_{n-1}^{k}$.

\section{SPONGY HYPERCUBES}

It is well-known that the Cartesian product of $n$ edges provides the hypercube: $\left(P_{2}\right)^{\square n}=Q_{n}$. Next, the Cartesian product of two hypercubes is another hypercube: $Q_{i} \square Q_{j}=Q_{i+j}$. 


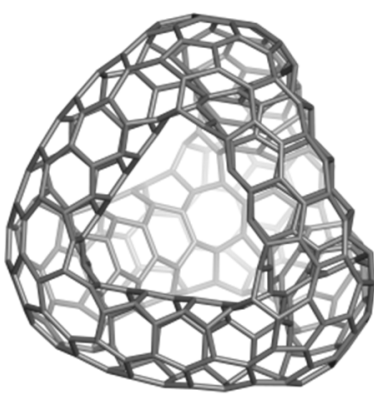

$\mathrm{H}_{340}$

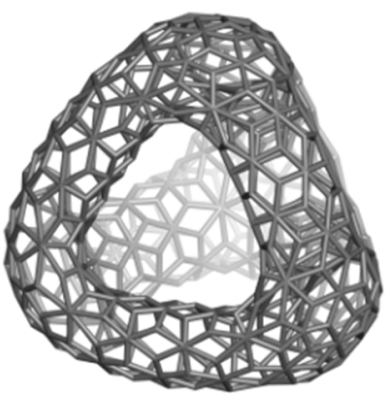

$d m\left(\mathrm{H}_{340}\right) \cdot 506\left\{4^{3} \cdot 4^{5} \cdot 4^{6} \cdot 4^{7}\right\}$

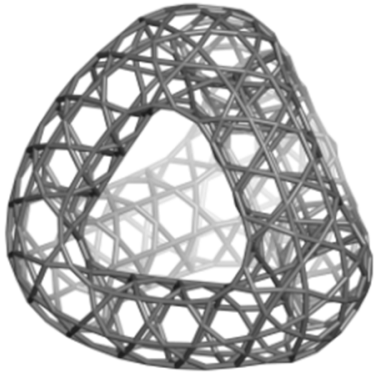

$m\left(\mathrm{H}_{340}\right) .510$

Figure 3. Operations acting on a triple torus (left): $o_{1}=d m$ (dual of medial, middle) and $o_{2}=m$ (right); vertex number differece: $\chi=-4 ; g=3$.

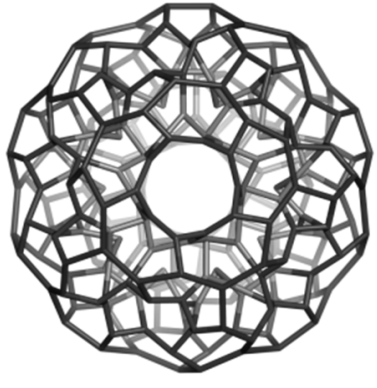

$d(s(\mathrm{D}) @ s(\mathrm{D}) 7 \mathrm{~S} .120) .280$

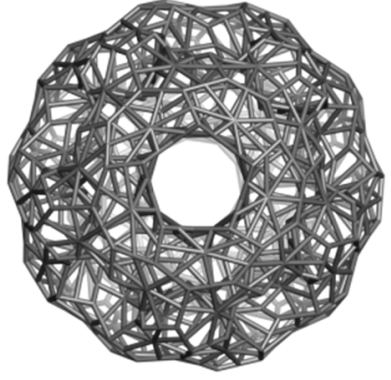

$p_{4}\left(C_{280}\right) .820$

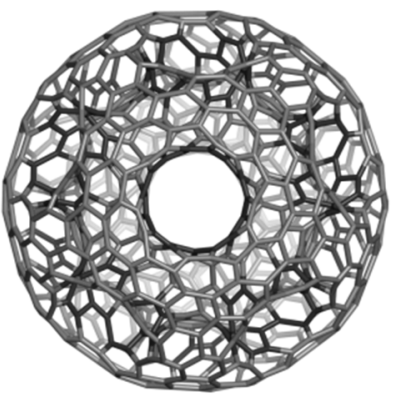

$/\left(C_{280}\right) .840$

Figure 4. Operations acting on a dodecahedral multi-torus (left): $o_{1}=p_{4}$ (middle) and $o_{2}=I$ (leapfrog, right); vertex number differece: $\chi=-20 ; g=11$.

Table 2. Figure count for the objects in Figures 2 to 4.

\begin{tabular}{|c|c|c|c|c|c|c|c|c|c|c|c|}
\hline Structure & $v$ & $e$ & $f_{3}$ & $f_{4}$ & $f_{5}$ & $f_{6}$ & $f_{7}$ & $f$ & $x$ & $g$ & Diff \\
\hline $\mathrm{H} 6.8$ & 48 & 96 & 0 & 48 & 0 & 0 & 0 & 48 & 0 & 1 & - \\
\hline$m(H 6.8)$ & 96 & 192 & 0 & 96 & 0 & 0 & 0 & 96 & 0 & 1 & - \\
\hline$d m(\mathrm{H} 6.8)$ & 96 & 192 & 0 & 96 & 0 & 0 & 0 & 96 & 0 & 1 & 0 \\
\hline $\mathrm{H}_{340}$ & 340 & 510 & 0 & 0 & 12 & 118 & 36 & 166 & -4 & 3 & - \\
\hline$d m\left(\mathrm{H}_{340}\right)$ & 506 & 1020 & 0 & 510 & 0 & 0 & 0 & 510 & -4 & 3 & - \\
\hline$m\left(\mathrm{H}_{340}\right)$ & 510 & 1020 & 340 & 0 & 12 & 118 & 36 & 506 & -4 & 3 & -4 \\
\hline $\mathrm{C}_{280}$ & 280 & 420 & 0 & 0 & 0 & 0 & 120 & 120 & -20 & 11 & - \\
\hline$p_{4}\left(C_{280}\right)$ & 820 & 1680 & 0 & 840 & 0 & 0 & 0 & 840 & -20 & 11 & - \\
\hline$/\left(C_{280}\right)$ & 840 & 1260 & 0 & 0 & 0 & 280 & 120 & 400 & -20 & 11 & -20 \\
\hline
\end{tabular}

Let now take the graph $G(d, v)$ of a $d$-connected polyhedron on $v$-vertices and make $n$-times the Cartesian product with an edge; the operation results in a "spongy hypercube" $G\left(d, v, Q_{n}\right)=G(d, v) \square^{n} P_{2}$.On each edge of the original polyhedral graph, a local hypercube $Q_{n}$ will evolve; these hypercubes are incident in a hypervertex, according to the original degree, $d$. It means that, in a spongy hypercube, the original 2-faces will not be counted. Figure 9 illustrates such a spongy hypercube, built on the fullerene $C_{60}\left(I_{h}\right)$.

Conjecture 4. The k-faces of a spongy hypercube $G\left(d, v, Q_{n}\right)$, built on a 3-polytope with vertices of degree $d$, are combinatorially counted from the previous rank faces; their alternating sum accounts for the genus of the embedding surface

$$
\begin{aligned}
G\left(d, v, Q_{n}, k\right)= & (v / n)[d \cdot n-(d-1)(n-k)] . \\
& 2^{(n-k-1)} \cdot\left(\begin{array}{l}
n \\
k
\end{array}\right) ; n>1 ; k=0,1, . . n .
\end{aligned}
$$

$\sum_{k=0}^{n}(-1)^{k} f_{k}=\chi(M)=2(1-g) ; n>1 ; k=0,1, . . n$ 


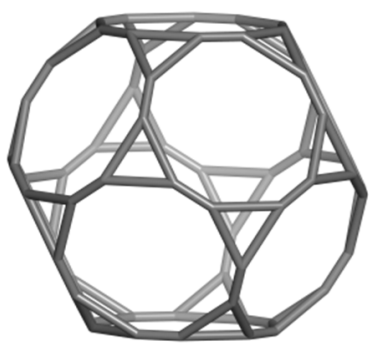

$t(\mathrm{D}) .60$

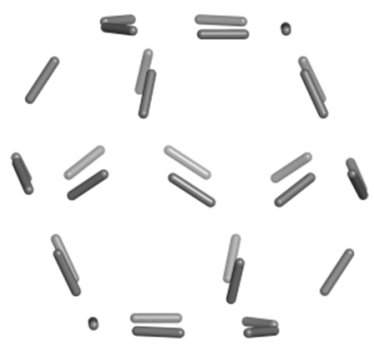

$M(D)$

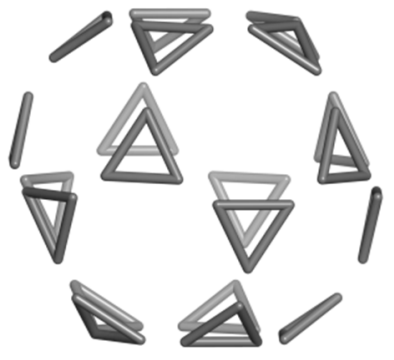

$C(D)$

Figure 5. Truncation of the dodecahedron D.

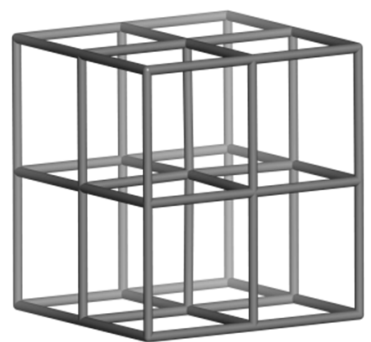

$\mathrm{RS}=4^{\wedge} 12 ;|\{27\}|$ deg $=6$;

$\mathrm{RSI}=12$

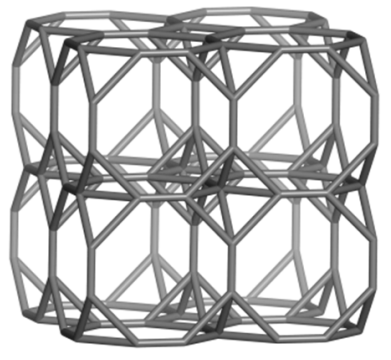

$\mathrm{RS}=3^{\wedge} 4.8^{\wedge} 4 ;|\{108\}|$ deg $=5$

$$
\mathrm{RSI}=4
$$

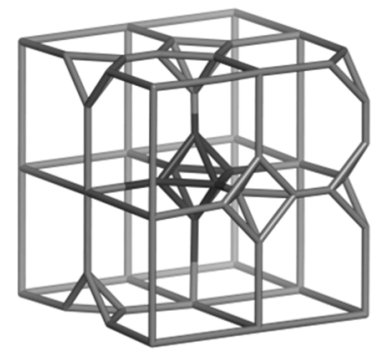

$|V|=55 ; 39$ atom types $\mathrm{RSI}=0.0663145$

Figure 6. Truncation of the cubic net (left): fully truncated (middle) and with defects (right).

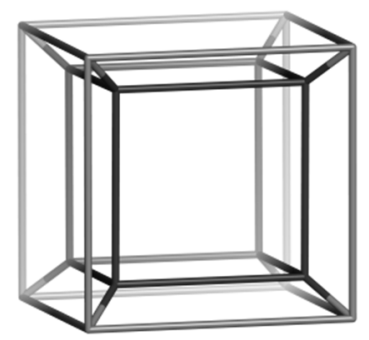

Q4.16

Figure 7. Truncation of the hypercube $Q_{n}$.

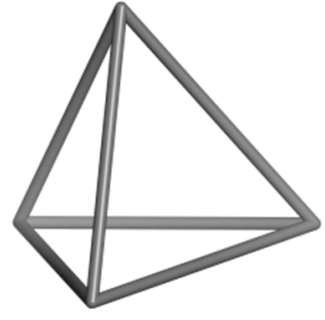

$\mathrm{S}_{3} .4$

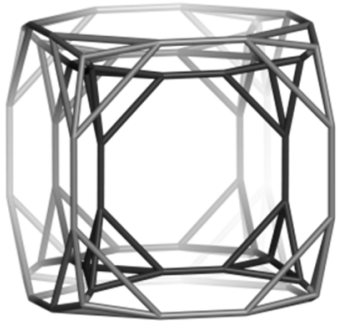

$t\left(Q_{4}\right) .64$

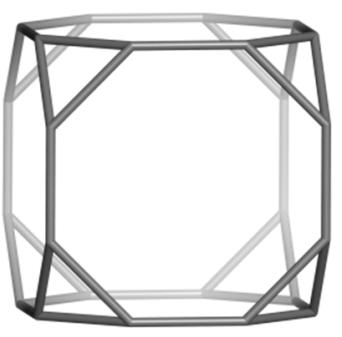

$t\left(Q_{3}\right) .24$

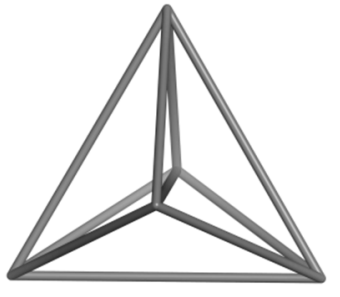

$\mathrm{S}_{4} .5$

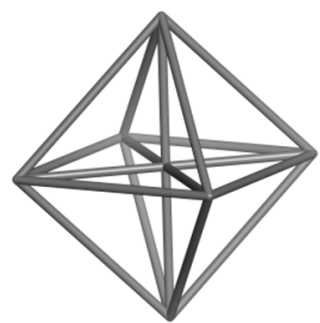

$\mathrm{S}_{5} 6$

Figure 8. Truncation of hypercube $Q_{n}$ : simplices $S_{n-1}$ substructures.

Formula (11) represents the "embedding" of the hypercube on any $d$-polyhedral cage ${ }^{[26]}$ (see the factor in the front of the almost classical hypercube counting), which transforms that cage in a hyper-multi-torus. Formula (12) gives the Euler characteristic, as a function of genus; in the graphs of Platonic solids, $g=f_{w}(G) / 2$, with $f_{w}$ being the 
Table 3. Figure count in truncated hypercube $Q_{n}$.

\begin{tabular}{|c|c|c|c|c|c|c|c|c|c|c|}
\hline$n$ & $v$ & $e$ & - & - & $4(2)$ & 3 & 4 & 5 & 6 & $\chi$ \\
\hline 3 & 8 & 12 & - & - & 6 & 0 & 0 & 0 & 0 & 2 \\
\hline 4 & 16 & 32 & - & - & 24 & 8 & 0 & 0 & 0 & 0 \\
\hline 5 & 32 & 80 & - & - & 80 & 40 & 10 & 0 & 0 & 2 \\
\hline 6 & 64 & 192 & - & - & 240 & 160 & 60 & 12 & 0 & 0 \\
\hline 7 & 128 & 448 & - & - & 672 & 560 & 280 & 84 & 14 & 2 \\
\hline$t\left(Q_{n}\right)$ & $n \times v\left(Q_{n}\right)$ & $n \times e\left(Q_{n}\right)$ & & & & & & & & \\
\hline$n$ & $v$ & $e$ & $3(2)$ & $8(2)$ & 2 & 3 & 4 & 5 & 6 & $\chi$ \\
\hline 3 & 24 & 36 & 8 & 6 & 14 & - & - & - & - & 2 \\
\hline 4 & 64 & 128 & 64 & 24 & 88 & 24 & - & - & - & 0 \\
\hline 5 & 160 & 400 & 320 & 80 & 400 & 200 & 42 & - & - & 2 \\
\hline 6 & 384 & 1152 & 1280 & 240 & 1520 & 1120 & 444 & 76 & - & 0 \\
\hline 7 & 896 & 3136 & 4480 & 672 & 5152 & 5040 & 2968 & 980 & 142 & 2 \\
\hline
\end{tabular}

Table 4. Figure count for spongy cubic $C_{8}\left(Q_{n}\right)$ hypercube $(g=3)$.

\begin{tabular}{|c|c|c|c|c|c|c|c|}
\hline$C_{8}\left(Q_{n}\right) \backslash k$ & 0 & 1 & 2 & 3 & 4 & 5 & $x$ \\
\hline$Q_{3}$ & 8 & 12 & 6 & 0 & 0 & 0 & 2 \\
\hline$Q_{4}$ & 16 & 32 & 24 & 8 & 0 & 0 & 0 \\
\hline$Q_{5}$ & 32 & 80 & 80 & 40 & 10 & 0 & 2 \\
\hline$Q_{6}$ & 64 & 192 & 240 & 160 & 60 & 12 & 0 \\
\hline$Q_{7}$ & 128 & 448 & 672 & 560 & 280 & 84 & 2 \\
\hline$C_{8}\left(Q_{1}\right) 3$ & 8 & 12 & 0 & 0 & 0 & 0 & -4 \\
\hline$C_{8}\left(Q_{2}\right) 4$ & 16 & 32 & 12 & 0 & 0 & 0 & -4 \\
\hline $\mathrm{C}_{8}\left(Q_{3}\right) 5$ & 32 & 80 & 56 & 12 & 0 & 0 & -4 \\
\hline$C_{8}\left(Q_{4}\right) 6$ & 64 & 192 & 192 & 80 & 12 & 0 & -4 \\
\hline$C_{8}\left(Q_{5}\right) 7$ & 128 & 448 & 576 & 352 & 104 & 12 & -4 \\
\hline
\end{tabular}

Table 5. Figure count for spongy dodecahedral $C_{20}\left(Q_{n}\right)$ hypercube $(g=6)$.

\begin{tabular}{cccccccc}
\hline$C_{20}\left(Q_{n}\right) \backslash k$ & 0 & 1 & 2 & 3 & 4 & 0 \\
\hline$\left(Q_{1}\right) 3$ & 20 & 30 & 0 & 0 & 0 & 0 & 0 \\
$\left(Q_{2}\right) 4$ & 40 & 80 & 30 & 0 & 30 & 0 & 0 \\
$\left(Q_{3}\right) 5$ & 80 & 200 & 140 & 480 & 200 & 30 & 0 \\
$\left(Q_{4}\right) 6$ & 160 & 480 & 1440 & 880 & 260 & 0 \\
$\left(Q_{5}\right) 7$ & 320 & 1120 & & & -10 & 30 \\
\hline
\end{tabular}

"window" faces of the polyhedron (embedded in an oriented surface). Formula (12) expresses the "spongy" character of these structures by the genus $g$ of the hypersurface. Note that (11) ignores the (hyper-) prisms evolved on each $f_{2}$ face of the original cage. In this respect, the case of full-hypercube is compared to the case of spongy-one (Table 4).

Formulas (11) and (12) are confirmed by the data listed in Tables 4 to 9. Figures and calculations were done by our original software programs CVNET ${ }^{[27]}$ and Nano Studio. ${ }^{[28]}$

\section{CONCLUSIONS}

Euler characteristic is a topological invariant that describes the shape or structure of polyhedra, or polytopes, in general. Counting polyhedral graph figures is directly related to

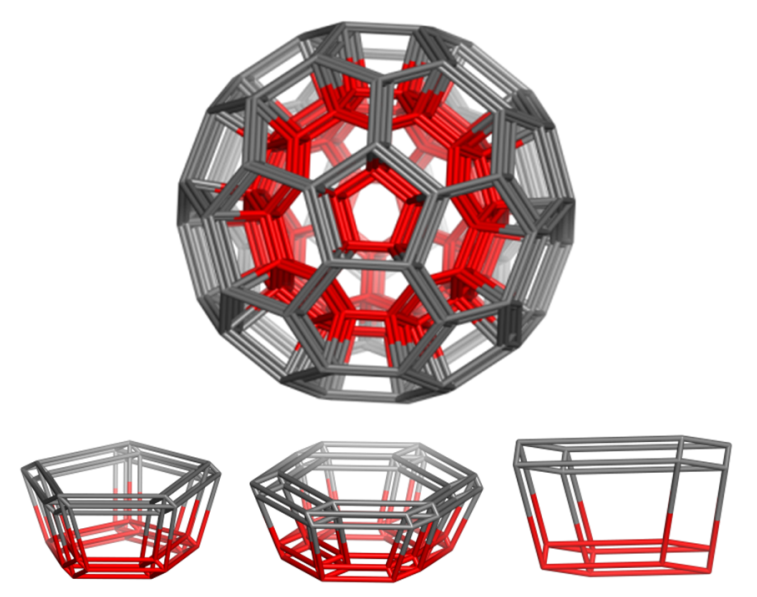

Figure 9. Spongy C60(Q4).480 (top), with its hyper-faces (bottom, left and middle) and a hyper-edge (bottom, right). 
Table 6. Figure count for spongy tetrahedral $C_{4}\left(Q_{n}\right)$ hypercube $(g=2)$.

\begin{tabular}{cccccccc}
\hline$C_{4}\left(Q_{n}\right) \backslash k$ & 0 & 1 & 2 & 3 & 4 & 0 \\
\hline$\left(Q_{1}\right) 3$ & 4 & 6 & 0 & 0 & 0 & 0 \\
$\left(Q_{2}\right) 4$ & 8 & 16 & 6 & 0 & 0 & 0 \\
$\left(Q_{3}\right) 5$ & 16 & 40 & 28 & 6 & 0 & -2 & 0 \\
$\left(Q_{4}\right) 6$ & 32 & 96 & 96 & 40 & 6 & 0 \\
$\left(Q_{5}\right) 7$ & 64 & 224 & 288 & 176 & 52 & -2 \\
\hline
\end{tabular}

Table 7. Figure count for spongy icosahedral $C_{12}\left(Q_{n}\right)$ hypercube $(g=10)$.

\begin{tabular}{cccccccc}
\hline$C_{12}\left(Q_{n}\right) \backslash k$ & 0 & 1 & 2 & 3 & 4 & 0 \\
\hline$\left(Q_{1}\right) 3$ & 12 & 30 & 0 & 0 & 0 & 0 & -18 \\
$\left(Q_{2}\right) 4$ & 24 & 72 & 30 & 0 & 30 & 0 & 0 \\
$\left(Q_{3}\right) 5$ & 48 & 168 & 132 & 432 & 192 & 30 & 0 \\
$\left(Q_{4}\right) 6$ & 96 & 384 & 1248 & 816 & 252 & 0 \\
$\left(Q_{5}\right) 7$ & 192 & 864 & & -18 & 30 \\
\hline
\end{tabular}

Table 8. Figure count for spongy octahedral $C_{6}\left(Q_{n}\right)$ hypercube $(g=4)$.

\begin{tabular}{cccccccc}
\hline$C_{6}\left(Q_{n}\right) \backslash k$ & 0 & 1 & 2 & 3 & 4 & 0 \\
\hline$\left(Q_{1}\right) 3$ & 6 & 12 & 0 & 0 & 0 & 0 & 0 \\
$\left(Q_{2}\right) 4$ & 12 & 30 & 12 & 0 & 12 & 0 & 0 \\
$\left(Q_{3}\right) 5$ & 24 & 72 & 54 & 180 & 78 & 12 & 0 \\
$\left(Q_{4}\right) 6$ & 48 & 168 & 528 & 336 & 102 & 0 \\
$\left(Q_{5}\right) 7$ & 96 & 384 & & -6 & -6 & 12 \\
\hline
\end{tabular}

Table 9. Figure count for spongy $C_{60}\left(Q_{n}\right)$ hypercube $(g=16)$.

\begin{tabular}{cccccccc}
\hline$C_{60}\left(Q_{n}\right) \backslash k$ & 0 & 1 & 2 & 3 & 4 & 0 \\
\hline$\left(Q_{1}\right) 3$ & 60 & 90 & 0 & 0 & 0 & 0 & -30 \\
$\left(Q_{2}\right) 4$ & 120 & 240 & 90 & 0 & 90 & 0 & 0 \\
$\left(Q_{3}\right) 5$ & 240 & 600 & 420 & 1440 & 600 & 90 & 0 \\
$\left(Q_{4}\right) 6$ & 480 & 1440 & 4320 & 2640 & 780 & 0 \\
$\left(Q_{5}\right) 7$ & 960 & 3360 & & & -30 & 90 \\
\hline
\end{tabular}

Euler characteristic. In this paper, it was shown the involvement of Euler characteristic in figure counting of polyhedral graphs designed by operations on maps. In this respect, pairs of operations were identified; it was shown that the difference of vertex number of the transformed graphs provide the Euler characteristic. The truncation operation and its use in transforming the cubic crystal network and the hypercube were detailed. Spongy hypercubes were built up by embedding the hypercube in polyhedral graphs, on which Euler characteristic was calculated by a general combinatorial formula, accounting for the "spongy" character of such structures. Concluding, the Euler characteristic is a theoretical tool, particularly useful in chemistry and crystallography to check the consistency of an assumed structure.

Acknowledgment. This work was supported by a grant of the Romanian National Authority for Scientific Research and Innovation, CCCDI - UEFISCDI, project number 8/2015, acronym GEMNS (under the frame of the ERA-NET EuroNanoMed II European Innovative Research and Technological Development Projects in Nanomedicine).

\section{REFERENCES}

[1] L. Euler, Novi comment acad. sc. imp. Petropol., 1752-3, 4, 109.

[2] D. Epstein, Euler's Formula References, The Geometry Junkyard, Theory Group, ICS, UC Irvine.

https://www.ics.uci.edu/ eppstein/junkyard/euler/ refs.html.

[3] I. Lakatos, Proofs and Refutations: The Logic of Mathematical Discovery, Cambridge Technol. Press, Cambrige, 1976.

[4] A. M. Legendre, Élements de géométrie, Paris, 1794.

[5] T. Leinster, Documenta Mathematica, 2008, 13, 21.

[6] D. M. Y. Sommerville, An Introduction to the Geometry of N Dimensions, Dover 1958.

[7] D. S. Richeson, Euler's Gem: The Polyhedron Formula and the Birth of Topology, Princeton University Press, 2008.

[8] O. Bonnet, C. R. Acad. Sci. Paris, 1853, 37, 529.

[9] R. Descartes, Progymnasmata de solidorum elementis, in Oeuvres de Descartes, vol. X, 265-276. 
[10] Y. Higuchi, J. Graph Theory, 2001, 38, 220.

[11] D. J. Klein, Phys. Chem. Chem. Phys. 2002, 4, 2099.

[12] J. H. C. Whitehead, Bull. Amer. Math. Soc. 1949, 55(5), 213.

[13] J. H. C. Whitehead, Bull. Amer. Math. Soc.1949, 55(3), 453.

[14] N. W. Johnson, The Theory of Uniform Polytopes and Honeycombs, Ph. D. Dissertation, University of Toronto, 1966.

[15] T. Pisanski, M. Randić, Geometry at Work. MAA Notes, 2000, 53, 174.

[16] J. H. Conway, H. Burgiel, C. Goodman-Strauss, The symmetries of things, A K Peters, Wellesley, Massachusetts, 2008.

[17] L. Schläfli, Theorie der vielfachen Kontinuität Zürcher und Furrer, Zürich, 1901 (Reprinted in: Ludwig Schläfli, 18141895, Gesammelte Mathematische Abhandlungen, Band 1, 167-387, Verlag Birkhäuser, Basel, 1950).

[18] H.S.M. Coxeter, Regular complex polytopes, Section 4.3 Flags and Orthoschemes, Section 11.3 Petrie polygons, 1974.

[19] V. Eberhard, Zur Morphologie der polyeder. B. G. Teubner, Leipzig, 1891.

[20] P. W. Fowler, Chem. Phys. Lett. 1986, 131, 444.

[21] M. V. Diudea, C. L. Nagy, Periodic nanostructures, Springer, Dordrecht, 2007.

[22] M. V. Diudea, V. R. Rosenfeld, J. Math. Chem. 2017, 55, 1014.

[23] Csaba L. Nagy, Mircea V. Diudea, MATCH Commun. Math. Comput. Chem., 2017, 77(2), 479.

[24] H. S. M. Coxeter, Regular Polytopes, $3^{\text {rd }}$ ed. Dover, 1973.

[25] P. McMullen, E. Schulte, Abstract Regular Polytopes, $1^{\text {st }}$ ed., Cambridge Univ. Press, Cambridge, 2002.

[26] M. V. Diudea, Multi-shell polyhedral clusters, Springer, Berlin, 2016 (in preparation).

[27] M. Stefu, M. V. Diudea, CageVersatile_CVNET, BabesBolyai Univ. Cluj, 2005.

[28] Cs. L. Nagy, M. V. Diudea, NANO-Studio, BabesBolyai Univ. Cluj, 2009. 